\title{
Cerebral Vasculitis in Henoch-Schönlein Purpura: A Case Report
}

\author{
Meltem AKÇABOY, Kibriya FİDAN, Yaşar KANDUR, Emel ISIYEL, Bahar BÜYÜKKARAGÖZ, \\ Sevcan Azime BAKKALOĞLU EZGÜ, Necla BUYAN, Enver HASANOĞLU, Oğuz SÖYLEMEZOĞLU \\ Department of Pediatric Nephrology, Medical Faculty of Gazi University, Ankara, Turkey
}

\begin{abstract}
Henoch-Schönlein purpura is a common form of systemic small vessel vasculitis in childhood. Although headache and behavioral changes have been described in a significant proportion of children with Henoch-Schönlein purpura, severe neurological complications are rare. In this article, we report a case of central vasculitis in a four-year-old boy who presented with hemiplegia and aphasia. The treatment options for cerebral vasculitis of Henoch-Schönlein purpura are numerous but controversial in pediatric patients. Our patient was successfully treated by pulse methylprednisolone and pulse cyclophosphamide. The patient was followed-up for four years without any sequel.

Keywords: Central vasculitis; Henoch-Schönlein purpura; treatment.
\end{abstract}

Henoch-Schönlein purpura (HSP) is the most common form of systemic small vessel vasculitis involving the skin, joints, kidneys, and gastrointestinal tract in childhood. ${ }^{1}$ Because of its systemic nature, involvement of other organ systems such as genitourinary, cardiopulmonary, and nervous systems may be observed..$^{2-4}$ Although headache and behavioral changes have been described in a significant proportion of children with HSP, severe neurological complications have been reported as rarely as $2 \%$ during the acute phase of the disease. . $^{4-6}$

In this article, we report a severe neurologic involvement in a four-year-old boy with HSP who was treated with intravenous pulse methylprednisolone and pulse cyclophosphamide, and followed-up for four years without any sequel.

\section{CASE REPORT}

A four-year-old boy presented with complaints of sudden onset of purpuric rash on his extensor surfaces of the lower extremities and arthralgia of the ankles for three days which had developed following a mild upper respiratory tract infection three days prior to admission. His medical and family histories were unremarkable. Physical examination showed a temperature of $36.6{ }^{\circ} \mathrm{C}$, respiratory rate of $28 /$ minute, pulse rate of $88 /$ minute, and blood pressure of $110 / 80 \mathrm{mmHg}$. Physical examination revealed symmetric palpable rash on his lower extremities. Both ankles were tender and swollen. Abdominal palpation and digital rectal examination were normal. Neurologic examination was also normal. Laboratory examination disclosed the following results: hemoglobin, $12.6 \mathrm{~g} / \mathrm{dL}$; hematocrit, $35 \%$; white blood cell count, $9,300 / \mathrm{mm}^{3}$; platelet count, $387,000 / \mathrm{mm}^{3}$; erythrocyte sedimentation rate, $17 \mathrm{~mm} /$ hour; C-reactive protein, $18.3 \mathrm{mg} / \mathrm{dL}$; while blood chemistry analysis was normal. Antistreptolysin $\mathrm{O}$ titer was 250 Todd units. Coagulation parameters, serum complements, and immunoglobulin levels were normal. Hepatitis markers, antinuclear antibody,

Received: September 26, 2016 Accepted: October 25, 2016 Published online: March 24, 2017

Correspondence: Meltem Akçaboy, MD. Gazi Üniversitesi Tıp Fakültesi Çocuk Nefrolojisi Bilim Dalı, 06500 Beşevler, Ankara, Turkey.

Tel: +90 312 - 2025230 e-mail: meltemileri@yahoo.com

(2017 Turkish League Against Rheumatism. All rights reserved. 


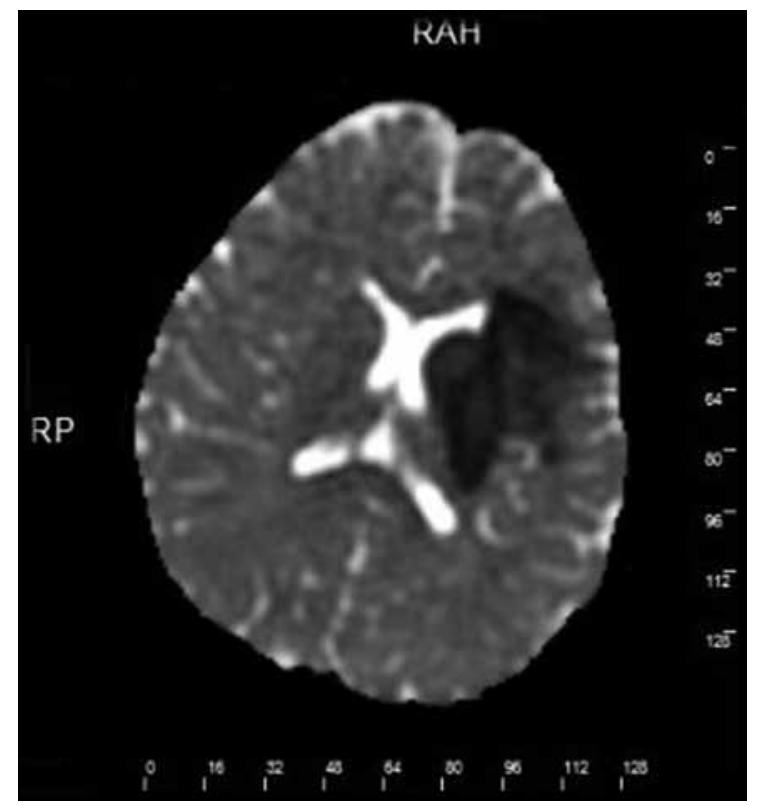

Figure 1. $\mathrm{T}_{2}$-weighed axial images (1.5-T) demonstrate multiple, high signal intensity areas in both cortical and subcortical areas of left parietal lobe.

and antineutrophil cytoplasmic antibody were negative. Urinalysis and the stool were normal.

Based on these clinical findings, the patient was diagnosed as HSP, and the skin biopsy showed leukocytoclastic vasculitis with histopathologic

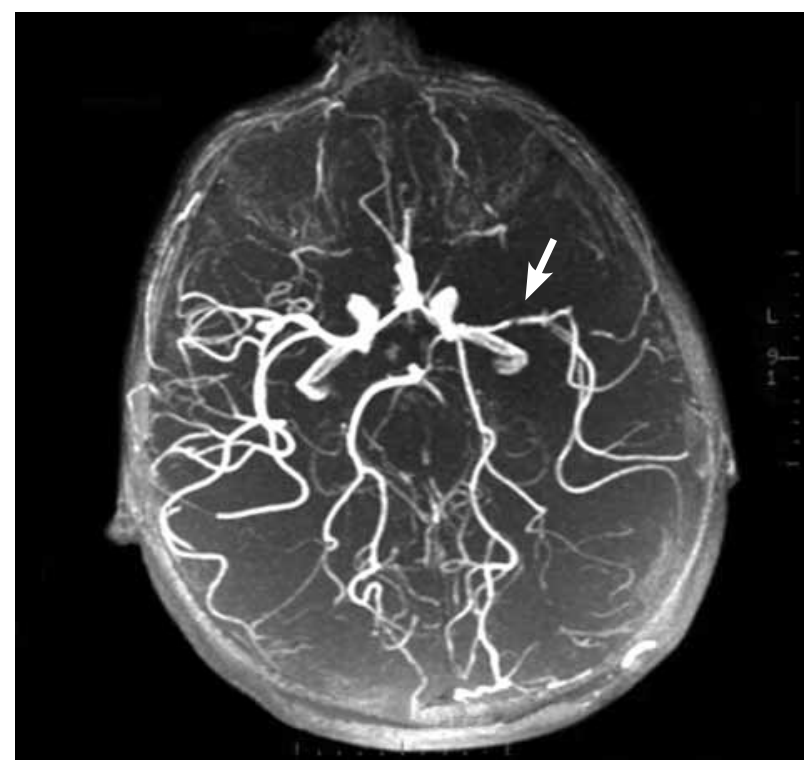

Figure 2. Initial magnetic resonance angiography reveals narrowing and irregularities in lumen of left middle cerebral artery (arrow). evidence of immunoglobulin (Ig) A and $\mathrm{M}$ deposition based on direct immunofluorescence staining. A written informed consent was obtained from the parent of patient. The patient was being followed-up as an out-patient. On the $15^{\text {th }}$ day of his follow-up, he admitted to the emergency department by sudden focal tonicclonic convulsions and unconsciousness. He was not able to use his right hand and foot, also he could not talk. His blood pressure was $105 / 70 \mathrm{mmHg}$. Fundoscopic examination was unremarkable. The physical examination showed aphasia, right sided central facial paralysis, and hemiplegia on the right. Deep tendon reflexes were exaggerated and there was extensor plantar response on the right side. Cerebellar functions and sensory testing were normal. In acute laboratory evaluation, serum electrolytes and glucose levels were normal. Immediate 1.5 Tesla magnetic resonance imaging (Magnetom Avanto, Siemens Medical Solutions, Erlangen, Germany) demonstrated multiple, high signal intensity areas on $\mathrm{T}_{2}$-weighted images in both cortical and subcortical areas of the left parietal lobe (Figure 1). Magnetic resonance angiography revealed narrowing and irregularities in the lumen of left middle cerebral artery resembling vasculitis (Figure 2). Electroencephalography showed diffuse generalized delta slow waves of moderate

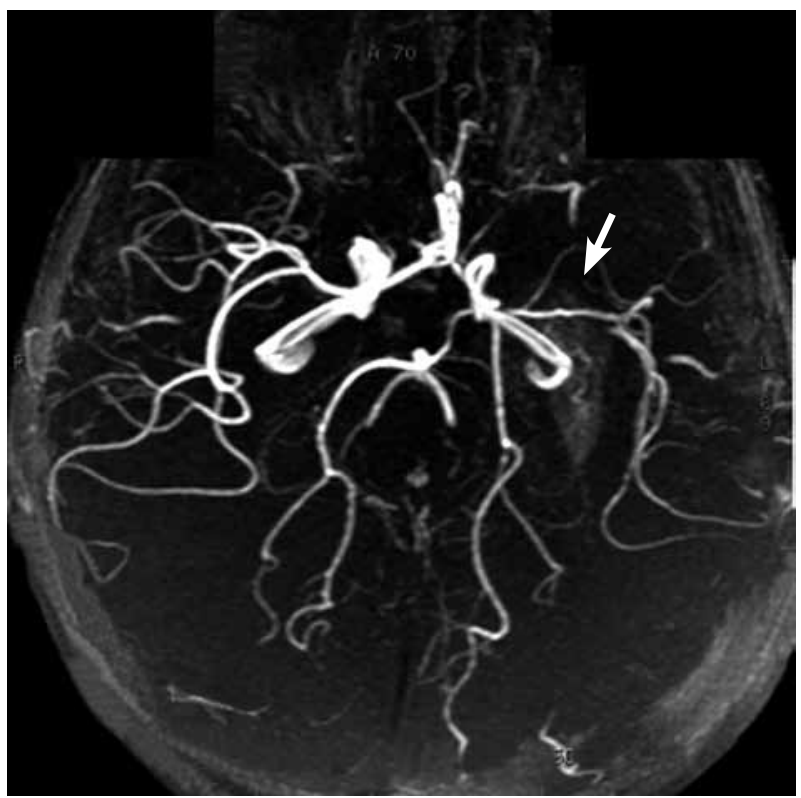

Figure 3. Follow-up magnetic resonance angiography shows significant improvement of cerebral lesions (arrow). 
amplitude without epileptiform discharges. Renal angiography and mesenteric vasculature were normal. The patient was diagnosed as cerebral vasculitis due to HSP. Intravenous pulse methylprednisolone (30 mg/kg/day) was given for three consecutive days; subsequently the patient received oral steroids (2 mg/kg/day) for cerebral vasculitis. Because of the unchanged clinical status of the patient, pulse cyclophosphamide therapy was begun. Patient received $500 \mathrm{mg} / \mathrm{m}^{2} /$ day pulse cyclophosphamide monthly for three months. He showed clinical improvement after cyclophosphamide therapy. The follow-up magnetic resonance angiography, performed 15 days after the initial one, demonstrated substantial improvement of cerebral lesions (Figure 3). His steroid treatment was stopped by tapering within six months. He had speech therapy and also physical rehabilitation and therapy for two years. He has been followed-up without any sequel for four years.

\section{DISCUSSION}

A great majority of patients recover from HSP completely, nevertheless, the condition may be associated with several rare complications. Although headache and behavioral changes are seen in many patients, severe neurological complications such as seizures, intracerebral hemorrhage, hemiplegia, and encephalopathy have also been reported albeit rarely during the course of HSP. ${ }^{1,2}$ Polyarteritis nodosa should be considered as a possible diagnosis in patients with HSP who have an unusual course, especially in patients presenting with severe neurological complications. Our patient admitted with hemiplegia and polyarteritis nodosa was ruled out with renal angiography. The diagnosis of cerebral vasculitis may be difficult since the underlying neurologic abnormalities in HSP may have a multifactorial etiology. It has been reported that concomitant occurrence of hypertension, uremia, metabolic abnormalities and, corticosteroid treatment might contribute to the neurologic manifestations. ${ }^{7}$ Our patient was normotensive and his serum chemistry was normal. Therefore, we could rule out the possibility of uremic and hypertensive encephalopathy. Garzoni et al. ${ }^{4}$ reported that without severe hypertension, the most frequent neurologic manifestations of patients with HSP are altered level of consciousness in 54\%, convulsions in $40 \%$, focal neurologic deficits in $26 \%$, visual abnormalities in $22 \%$, and verbal disability in $10 \%$ of patients. Our patient admitted with hemiplegia and aphasia together. Neuroimaging results of cerebral lesions with central vasculitis in patients with HSP have been reported to be normal in 14\%. The most frequently reported lesions were ischemic in $43 \%$ and hemorrhagic in 33\% of the patients. ${ }^{7,8}$ Ischemic lesions are usually multiple, but if there is a single vascular cerebral ischemia, the middle cerebral artery is mostly affected, like in our patient. A review of the literature indicates that neurologic symptoms usually develop less than two weeks after the onset of HSP. ${ }^{8}$ Our patient was diagnosed on the $15^{\text {th }}$ day of the disease.

Recently, the correlation of renal involvement with the presence of serum pentraxin 3 levels and IgM depositions accompanying IgA depositions in skin biopsies of patients with HSP was shown. ${ }^{9}$ Particularly, the presence of $\operatorname{IgM}$ deposition showed significant association with subsequent renal involvement. Our patient's skin biopsy showed leukocytoclastic vasculitis with histopathologic evidence of $\operatorname{Ig} A$ and $\operatorname{IgM}$ deposition based on direct immunofluorescence staining but renal involvement was absent. The deposition of IgM should predict not only the renal complications but also the cranial nerve involvement in patients with HSP.

Henoch-Schönlein purpura is usually a self-limited disease. ${ }^{2-4}$ However, the potential morbidity and mortality associated with central nervous system involvement have led to aggressive treatment. The treatment of cerebral vasculitis in HSP is based on anecdotal reports. Pulse corticosteroid therapy is widely used as the first-line treatment and has proven to be effective in most patients. ${ }^{3-4,7}$ For patients who show corticosteroid dependency or who do not respond to the therapy, other immunosuppressive drugs such as cyclosporine A, cyclophosphamide, or mycophenolate mofetil have been used by patient-based decisions. ${ }^{7,8}$ Another treatment option for neurologic involvement in HSP is plasmapheresis. ${ }^{8,10}$ Plasmapheresis is thought to primarily modulate the immune response by eliminating and diminishing plasma immunological mediators that are responsible for HSP. ${ }^{10}$ Like in severe Henoch-Schönlein 
glomerulonephritis, combined therapy with corticosteroids and cyclophosphamide may be appropriate in a patient with relevant ischemic cerebral lesions and HSP. ${ }^{7}$ Our patient was successfully treated with the combined therapy of pulse methylprednisolone and pulse cyclophosphamide followed by long-term corticosteroid treatment.

In conclusion, cerebral vasculitis should be suspected in all cases of HSP with neurologic manifestations. Magnetic resonance angiography is the modality of choice for diagnosis and follow-up evaluation. Although treatment options are numerous and controversial, combined therapy with pulse corticosteroid and pulse cyclophosphamide should be the first choice in pediatric patients.

\section{Declaration of conflicting interests}

The authors declared no conflicts of interest with respect to the authorship and/or publication of this article.

\section{Funding}

The authors received no financial support for the research and/or authorship of this article.

\section{REFERENCES}

1. Weiss PF. Pediatric vasculitis. Pediatr Clin North Am 2012;59:407-23.
2. Deng F, Lu L, Zhang Q, Hu B, Wang SJ, Huang N. Henoch-Schönlein purpura in childhood: treatment and prognosis. Analysis of 425 cases over a 5 -year period. Clin Rheumatol 2010;29:369-74.

3. Bakkaloğlu SA, Ekim M, Tümer N, Deda G, Erden I, Erdem T. Cerebral vasculitis in Henoch-Schönlein purpura. Nephrol Dial Transplant 2000;15:246-8.

4. Garzoni L, Vanoni F, Rizzi M, Simonetti GD, Goeggel Simonetti B, Ramelli GP, et al. Nervous system dysfunction in Henoch-Schonlein syndrome: systematic review of the literature. Rheumatology (Oxford) 2009;48:1524-9.

5. McCarthy HJ, Tizard EJ. Clinical practice: Diagnosis and management of Henoch-Schönlein purpura. Eur J Pediatr 2010;169:643-50.

6. Ostergaard JR, Storm K. Neurologic manifestations of Schönlein-Henoch purpura. Acta Paediatr Scand 1991;80:339-42.

7. Belman $\mathrm{AL}$, Leicher $\mathrm{CR}$, Moshé SL, Mezey AP. Neurologic manifestations of Schoenlein-Henoch purpura: report of three cases and review of the literature. Pediatrics 1985;75:687-92.

8. Eun SH, Kim SJ, Cho DS, Chung GH, Lee DY, Hwang PH. Cerebral vasculitis in Henoch-Schönlein purpura: MRI and MRA findings, treated with plasmapheresis alone. Pediatr Int 2003;45:484-7.

9. Yüksel S, Çağlar M, Eurengül H, Becerir T, Tepeli E, Ergin A, et al. Could serum pentraxin 3 levels and IgM deposition in skin biopsies predict subsequent renal involvement in children with Henoch-Schönlein purpura? Pediatr Nephrol 2015;30:969-74.

10. Wen YK, Yang Y, Chang CC. Cerebral vasculitis and intracerebral hemorrhage in Henoch-Schönlein purpura treated with plasmapheresis. Pediatr Nephrol 2005;20:223-5. 